\title{
Productions of $f_{1}(1420)$ in pion and kaon induced reactions
}

\author{
Xiao-Yun Wang, ${ }^{1, \dagger}$ Jun $\mathrm{He},{ }^{2, *}$ Quanjin Wang, ${ }^{3}$ and Hao $\mathrm{Xu}^{4}$ \\ ${ }^{1}$ Department of physics, Lanzhou University of Technology, Lanzhou 730050, China \\ ${ }^{2}$ Department of Physics and Institute of Theoretical Physics, Nanjing Normal University, \\ Nanjing, Jiangsu 210097, China \\ ${ }^{3}$ Lanzhou University of Technology, Lanzhou 730050, China \\ ${ }^{4}$ Department of Applied Physics, School of Science, Northwestern Polytechnical University, \\ Xi'an 710129, China
}

(Received 9 October 2018; published 15 January 2019)

\begin{abstract}
The $f_{1}(1420)$ productions via pion and kaon induced reactions on a proton target are investigated in an effective Lagrangian approach. Two treatments of the $t$-channel Born term, the Feynman model, and the Regge model are introduced to calculate the total and differential cross sections of the $\pi^{-} p \rightarrow f_{1}(1420) n$ and $K^{-} p \rightarrow f_{1}(1420) \Lambda$ reactions. The numerical results indicate that the experimental data of the total cross section of the $\pi^{-} p \rightarrow f_{1}(1420) n$ reaction can be reproduced in both the Feynman and the Regge models. Based on the parameters determined in the pion induced reaction, the cross sections of the $K^{-} p \rightarrow f_{1}(1420) \Lambda$ reaction, about which there is little data found in the literature, are predicted in the same beam momentum range. It is found that the line shapes of the total cross section obtained in a kaon induced reaction with two treatments are quite different. The cross sections for both reactions are at an order of magnitude of $\mu \mathrm{b}$, or larger, at a beam momenta up to $10 \mathrm{GeV} / \mathrm{c}$. The differential cross sections for both pion and kaon induced reactions are also present. It is found that in the Regge model, the $t$ channel provides a sharp increase at extreme forward angles. The results suggest that the experimental study of the $f_{1}(1420)$ in the kaon induced reaction on a proton target is as promising as in the pion induced reaction. Such an experimental measurement is also very helpful to clarify the production mechanism of the $f_{1}(1420)$.
\end{abstract}

DOI: 10.1103/PhysRevD.99.014020

\section{INTRODUCTION}

The study of light mesons is an important way to understand the nonperturbative QCD. Many light mesons have been observed and listed in the Review of Particle Physics (PDG) [1]. However, the internal structure of light mesons is still a confusing problem due to large nonperturbative effects in the light flavor sector. Currently, the electron-positron collision is the most important way to study light mesons. It will be very helpful to study the production and property of light mesons in different reactions. A new detector, glueX, was equipped at CEBAF after a $12 \mathrm{GeV}$ upgrade, which will focus on light meson spectroscopy [2]. Pion induced light meson production is very important in the history of the discovery of many light mesons. The secondary pion beam is accessible

\footnotetext{
* Corresponding author. junhe@njnu.edu.cn

xywang01@outlook.com

Published by the American Physical Society under the terms of the Creative Commons Attribution 4.0 International license. Further distribution of this work must maintain attribution to the author(s) and the published article's title, journal citation, and DOI. Funded by SCOAP ${ }^{3}$.
}

at J-PARC [3] and COMPASS [4] with high intensity. The kaon beam can be also used to study light mesons, and it is available at OKA@U-70 [5], SPS@CERN [6], and J-PARC [7]. The data from future experiments at those facilities will provide a good opportunity to deepen our understanding of the internal structure of light mesons.

In the PDG, the $f_{1}(1420)$ is listed as an axial-vector state with quantum numbers $I^{G}\left(J^{P C}\right)=0^{+}\left(1^{++}\right)$with a suggested mass of $1426.4 \pm 0.9 \mathrm{MeV}$ and a suggested width of $54.9 \pm 2.6 \mathrm{MeV}$ [1]. The $f_{1}(1420)$ meson was first observed in a pion-nucleon interaction in the Lawrence Radiation Laboratory in 1967 [8], and it was confirmed in other experiments with pion beams around the year 1980 [9-11]. The $f_{1}(1420)$ was also observed in recent experiments in $e^{+} e^{-}$and $J / \psi$ decays [12-14]. Though the $f_{1}(1420)$ is well established experimentally as a resonance structure, the internal structure of the $f_{1}(1420)$ was, until now, still unclear. In the conventional $q \bar{q}$ picture, the $f_{1}(1420)$ can be classified as a partner to the $f_{1}(1285)$ in the ${ }^{3} P_{1}$ nonet of axial mesons, and the mixture of nonstrange $f_{1 q}=(u \bar{u}+d \bar{d}) / \sqrt{2}$ and hidden-strange $f_{1 s}=s \bar{s}$ was also discussed in the literature [15-17]. However, a recent study in Ref. [18] suggested that the $f_{1}(1420)$ is not 
a genuine resonance but results from the decay modes of the $f_{1}(1285)$ in $K^{*} \bar{K}$ and $\pi a_{0}(980)$ channels.

To determine the origin of the $f_{1}(1420)$, more precise experimental data are required. In this work, based on the existent old data, we will analyze the $f_{1}(1420)$ production in pion induced reactions in an effective Lagrangian approach. The kaon induced production will be discussed based on the results of the pion induced interaction, which will be helpful for future high-precision experimental studies. Since in the current work we focus on the production mechanism of the $f_{1}(1420)$, the coupling constants are still determined with an assumption that the $f_{1}(1420)$ is a genuine resonance [1].

This paper is organized as follows: After the introduction, we present the formalism including Lagrangians and amplitudes of the $f_{1}(1420)$ productions in Sec. II. The numerical results of the cross section follow in Sec. III. Finally, the paper ends with a brief summary.

\section{FORMALISM}

The reaction mechanisms of pion and kaon induced productions of the $f_{1}(1420)$ are illustrated in Fig. 1. Usually, the contribution from the $s$ channel with a nucleon pole is expected to be very small, and it will be neglected in the current calculation. The $u$-channel contribution is usually small and negligible at low energies [19]. Considering that the experimental data points at high energies were obtained by the continuation of the $t$-channel contribution at very forward angles to all angles, it is reasonable to calculate the cross section with only the $t$-channel contribution. In the present work, we do not include the contributions from the nucleon resonances in the $s$ and $u$ channels.

Since the dominant decay of the $f(1420)$ is $K \bar{K}^{*}$, it is reasonable to take $K^{*}$ exchange as the dominant contribution in the $t$ channel of kaon induced production. For pion induced production, we need the vertex for decay of the $f_{1}(1420)$ with a pion. In Ref. [20], a branch ratio of about $5 \%$ was reported in the $a_{0} \pi$ channel. Hence, in the current work, we adopt $a_{0}$ exchange in the $t$ channel of pion induced production as in the case of pion induced $f_{1}(1285)$ production [19].

\section{A. Lagrangians}

For pion induced production of the $f_{1}(1420)$ to describe the $t$-channel $a_{0}(980) \quad\left(\equiv a_{0}\right)$ exchange, we need the following Lagrangians [21-24],
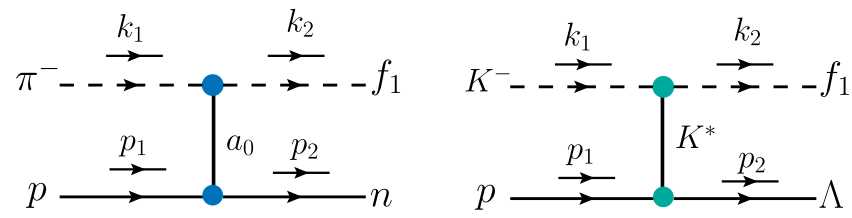

FIG. 1. Feynman diagrams for the $\pi^{-} p \rightarrow f_{1}(1420) n$ reaction (left) and for the $K^{-} p \rightarrow f_{1}(1420) \Lambda$ reaction (right).

$$
\begin{aligned}
\mathcal{L}_{a_{0} N N} & =g_{a_{0} N N} \bar{N}\left(\boldsymbol{\tau} \cdot \boldsymbol{a}_{0}\right) N, \\
\mathcal{L}_{f_{1} a_{0} \pi} & =-g_{f_{1} a_{0} \pi} f_{1}^{\mu} \partial_{\mu} \boldsymbol{\pi} \cdot \boldsymbol{a}_{0},
\end{aligned}
$$

where $N, f_{1}, a_{0}$ and $\pi$ are the nucleon, $f_{1}(1420), a_{0}(980)$, and $\pi$ meson fields, respectively. As suggested in the PDG [1], the average width of $f_{1}(1420)$ is $54.9 \mathrm{MeV}$. In addition, the branching fraction of $f_{1}(1420)$ decay to $a_{0} \pi$ was determined to be $4 \%$ in Ref. [20]. Thus, one gets $\Gamma_{f_{1} \rightarrow a_{0} \pi} \simeq 2.2 \mathrm{MeV}$, which leads to a value of coupling constant $g_{f_{1} a_{0} \pi} \simeq 2.72$. In order to reduce the number of the free parameter, we take the best fitting value $g_{a_{0} N N}=28.44$ in the pion induced $f_{1}(1285)$ production [19].

For kaon induced production, the relevant Lagrangians for the $t$ channel read [24-26],

$$
\mathcal{L}_{f_{1} K^{*} K}=\frac{g_{f_{1} K^{*} K}}{m_{f_{1}}}\left(\partial_{\mu} K_{\nu}^{*} \partial^{\mu} K f_{1}^{\nu}-\partial_{\mu} K_{\nu}^{*} \partial^{\nu} K f_{1}^{\mu}\right)
$$

$\mathcal{L}_{K^{*} N \Lambda}=-g_{K^{*} N \Lambda} \bar{N}\left(K^{*}-\frac{\kappa_{K^{*} N \Lambda}}{2 m_{N}} \sigma_{\mu \nu} \partial^{\nu} K^{* \mu}\right) \Lambda+$ H.c.,

where $m_{f_{1}}$ is the mass of $f_{1}$ meson, and $K, K^{*}, f_{1}, N$, and $\Lambda$ are the kaon, $K^{*}, f_{1}(1420), \Lambda$, and the nucleon fields, respectively. Here, we adopt the coupling constants $g_{K^{*} N \Lambda}=-4.26$ and $\kappa_{K^{*} N \Lambda}=2.66$ as suggested by the Nijmegen potential [27].

The value of $g_{f_{1} K^{*} K}$ can be determined from the decay width

$$
\begin{aligned}
\Gamma_{f_{1} \rightarrow K^{*} K}= & \left(\frac{g_{f_{1} K^{*} K}}{m_{f_{1}}}\right)^{2} \frac{\left|\vec{p}_{K}^{\text {c.m. }}\right|}{24 \pi m_{f_{1}}^{2}} \\
& \times\left[\frac{\left(m_{f_{1}}^{2}-m_{K^{*}}^{2}-m_{K}^{2}\right)^{2}}{2}+m_{K^{*}}^{2} E_{K}^{2}\right],
\end{aligned}
$$

with

$$
\begin{aligned}
\left|\vec{p}_{K}^{\text {c.m.m. }}\right| & =\frac{\lambda\left(m_{f_{1}}^{2}, m_{K^{*}}^{2}, m_{K}^{2}\right)}{2 m_{f_{1}}}, \\
E_{K} & =\sqrt{\left|\vec{p}_{K}^{\text {c.m. }}\right|^{2}+m_{K}^{2}},
\end{aligned}
$$

where $\lambda$ is the Källen function with a definition of $\lambda(x, y, z)=\sqrt{(x-y-z)^{2}-4 y z}$. Since the branching fraction of $f_{1}(1420)$ decay to $K^{*} K$ was suggested to be $96 \%$ in Ref. [20], one gets $g_{f_{1} K^{*} K} / m_{f_{1}} \simeq 8.36$ by taking $\Gamma_{f_{1} \rightarrow K^{*} K} \simeq 52.7 \mathrm{MeV}[1,20]$.

For the $t$-channel exchange [23], the form factor $F\left(q^{2}\right)=\left(\Lambda^{2}-m^{2}\right) /\left(\Lambda^{2}-q^{2}\right)$ is taken into account. Here, $q$ and $m$ are the four-momentum and mass of the exchanged meson, respectively. The value of the cutoff $\Lambda$ will be determined by fitting the experimental data. 


\section{B. Amplitudes}

According to the above Lagrangians, the scattering amplitude of the $\pi^{-} p \rightarrow f_{1}(1420) n$ or $K^{-} p \rightarrow f_{1}(1420) \Lambda$ reaction can be written as

$$
-i \mathcal{M}=\epsilon_{f_{1}}^{\mu *}\left(k_{2}\right) \bar{u}\left(p_{2}\right) \mathcal{A}_{\mu} u\left(p_{1}\right),
$$

where $\epsilon_{f_{1}}^{\mu *}$ is the polarization vector of $f_{1}$ meson, and $\bar{u}$ or $u$ is the Dirac spinor of nucleon or $\Lambda$ baryon.

For the $\pi^{-} p \rightarrow f_{1}(1420) n$ reaction, the reduced amplitude $\mathcal{A}_{i, \mu}$ reads

$$
\mathcal{A}_{\mu}^{\left(a_{0}\right)}=i \sqrt{2} g_{a_{0} N N} g_{f_{1} a_{0} \pi} F\left(q^{2}\right) \frac{1}{t-m_{a_{0}}^{2}} k_{1 \mu},
$$

where $t=\left(k_{1}-k_{2}\right)^{2}$ is the Mandelstam variables. The coupling constants are fixed with the experimental data and the fitting of the pion induced $f_{1}(1285)$ production as addressed above.

For the $K^{-} p \rightarrow f_{1}(1420) \Lambda$ reaction, the reduced amplitude $\mathcal{A}_{\mu}$ is written as

$$
\begin{aligned}
\mathcal{A}_{\mu}^{\left(K^{*}\right)}= & i g_{K^{*} N \Lambda} \frac{g_{f_{1} K^{*} K}}{m_{f_{1}}} F\left(q^{2}\right)\left(\gamma_{\xi}-i \frac{\kappa_{K^{*} N \Lambda}}{2 m_{N}} \gamma_{\xi} q_{K^{*}}\right) \\
& \times \frac{\mathcal{P}^{\nu \xi}}{t-m_{K^{*}}^{2}}\left[\left(k_{1}-k_{2}\right) \cdot k_{1} g_{\mu \nu}-\left(k_{1}-k_{2}\right)_{\mu} \cdot k_{1 \nu}\right],
\end{aligned}
$$

with

$$
\mathcal{P}^{\nu \xi}=i\left(g^{\nu \xi}+q_{K^{*}}^{\nu} q_{K^{*}}^{\xi} / m_{K^{*}}^{2}\right) .
$$

Here, the coupling constants are also fixed as in the pion induced production. Hence, the only free parameter is the cutoff in the form factor.

\section{Reggeized $t$-channel}

To analyze hadron production at high energies, a more economical approach may be provided by a Reggeized treatment [25,26,28-31]. In our previous works [19,29,32], an interpolating Reggeized treatment was introduced to interpolate the Regge trajectories smoothly to the Feynman propagator at low energies, as proposed in Ref. [33]. Because there are only 4 data points, we do not adopt the interpolating Reggeized treatment, but we discuss both the Feynman model and the Regge model. In the Feynman model, the $t$-channel amplitude in Eqs. (9) and (10) is applied directly. The Regge model can be introduced by replacing the $t$-channel Feynman propagator with the Regge propagator as,

$$
\frac{1}{t-m_{a_{0}}^{2}} \rightarrow\left(\frac{s}{s_{\text {scale }}}\right)^{\alpha_{a_{0}}(t)} \frac{\pi \alpha_{a_{0}}^{\prime}}{\Gamma\left[1+\alpha_{a_{0}}(t)\right] \sin \left[\pi \alpha_{a_{0}}(t)\right]},
$$

$\frac{1}{t-m_{K^{*}}^{2}} \rightarrow\left(\frac{s}{s_{\text {scale }}}\right)^{\alpha_{K^{*}}(t)-1} \frac{\pi \alpha_{K^{*}}^{\prime}}{\Gamma\left[\alpha_{K^{*}}(t)\right] \sin \left[\pi \alpha_{K^{*}}(t)\right]}$.

The scale factor $s_{\text {scale }}$ is fixed at $1 \mathrm{GeV}^{2}$. In addition, the Regge trajectories of $\alpha_{a_{0}}(t)$ and $\alpha_{K^{*}}(t)$ read as [30,31],

$$
\begin{aligned}
& \alpha_{a_{0}}(t)=-0.5+0.6 t / \mathrm{GeV}^{2}, \\
& \alpha_{K^{*}}(t)=1+0.85\left(t-m_{K^{*}}^{2}\right) / \mathrm{GeV}^{2} .
\end{aligned}
$$

After the Reggeized treatment is introduced, no additional parameter is introduced.

\section{NUMERICAL RESULTS}

With the preparation in the previous section, the cross section of the $\pi^{-} p \rightarrow f_{1}(1420) n$ and $K^{-} p \rightarrow f_{1}(1420) \Lambda$ reactions will be calculated and compared with the experimental data [8-11]. The differential cross section in the center of mass (c.m.) frame is written as

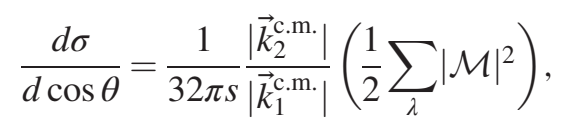

where $s=\left(k_{1}+p_{1}\right)^{2}$, and $\theta$ denotes the angle of the outgoing $f_{1}(1420)$ meson relative to the $\pi / K$ beam direction in the c.m. frame. $\vec{k}_{1}^{\text {c.m. }}$ and $\vec{k}_{2}^{\text {c.m. }}$ are the threemomenta of the initial $\pi / K$ beam and final $f_{1}(1420)$, respectively.

\section{A. Cross section of the $\pi^{-} p \rightarrow f_{1}(1420) n$ reaction}

In this work, we minimize $\chi^{2}$ per degree of freedom (d.o.f.) for the experimental data of the total cross section by fitting the cutoff parameter $\Lambda$ using a total of 4 data points at the beam momentum $p_{\text {Lab }}$ from 3.1 to $13.5 \mathrm{GeV} / \mathrm{c}$. The fitted cutoff and the $\chi^{2} /$ dof are listed in Table I.

From Fig. 2, it is found that the experimental data of the total cross section for the $\pi^{-} p \rightarrow f_{1}(1420) n$ reaction is reproduced in both the Feynman and the Regge models. The line shapes of total cross section in both models are analogous. The total cross section increases sharply near the threshold and reaches a maximum at a beam momentum of about $3 \mathrm{GeV} / \mathrm{c}$. The Regge model gives a cross section that is a little larger than the Feynman model in this momentum range. The total cross section decreases at momenta larger than $3 \mathrm{GeV} / \mathrm{c}$ in both the Feynman and the

TABLE I. The fitted value of free parameter $\Lambda_{t}$ in the unit of $\mathrm{GeV}$.

\begin{tabular}{lcc}
\hline \hline & $\Lambda$ & $\chi^{2} /$ dof \\
\hline Feynman & $1.18 \pm 0.01$ & 1.68 \\
Regge & $1.60 \pm 0.03$ & 1.93 \\
\hline \hline
\end{tabular}




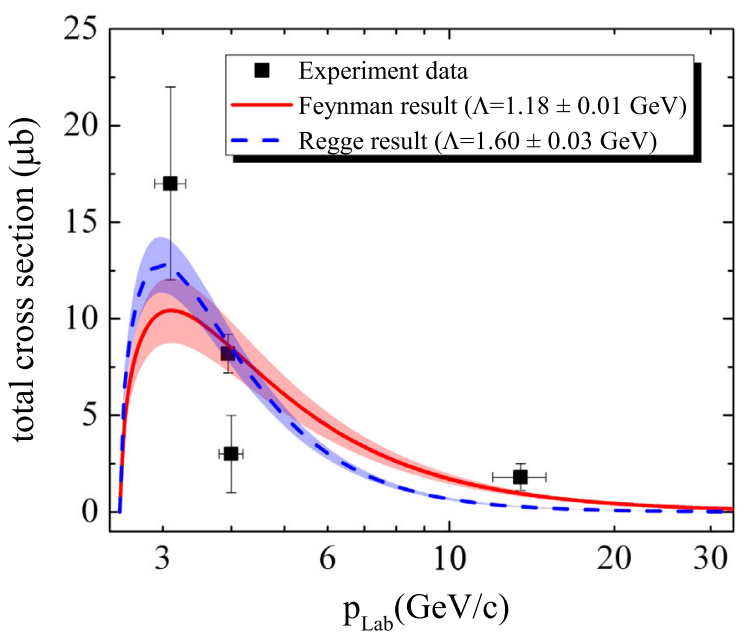

FIG. 2. Total cross section for the $\pi^{-} p \rightarrow f_{1}(1420) n$ reaction. The Full (red) and dashed (blue) lines are for the Feynman and Regge models, respectively. The bands stand for the error bar of the cutoff $\Lambda$. The experimental data are from Refs. [8-11].

Regge models. In this momentum range, the Feynman model gives a larger total cross section. The $\chi^{2} /$ dof are 1.68 and 1.93 for the Feynman and the Regge models, respectively. Both models can describe the existent rough data quantitatively. Based on the current results, it can be expected that the data will be described better if a mixing of the two models, that is, the interpolating Regge treatment $[19,29,32,33]$, is introduced, which requires more precise data. From Fig. 2, the $\chi^{2}$ comes mainly from the two data points around $4 \mathrm{GeV} / \mathrm{c}$. In both models, the upper data point is roughly obtained, which can be checked in future high-precision experiment.

In Fig. 3, we present our prediction of the differential cross section of the $\pi^{-} p \rightarrow f_{1}(1420) n$ reaction in two schemes at different beam momenta. It can be seen that the differences between the Regge and the Feynman models at low momenta are small but become large at higher momenta. With increase of the beam momentum, the slope of the curve in the Regge model is steeper than that in the Feynman model at forward angles, which can be tested by further experiment to clarify the role of the Reggeized treatment.

\section{B. Cross section of the $K^{-} p \rightarrow f_{1}(1420) \Lambda$ reaction}

Since there exists no experimental data for the $K^{-} p \rightarrow$ $f_{1}(1420) \Lambda$ reaction, here we give the prediction of the cross section for the $K^{-} p \rightarrow f_{1}(1420) \Lambda$ reaction. In Ref. [11], the experiment shows that $\sigma\left(K^{-} p \rightarrow f_{1}(1420) \Lambda\right) /$ $\sigma\left(\pi^{-} p \rightarrow f_{1}(1420) n\right)>10$ at a beam momentum $p_{\mathrm{Lab}}=$ $32.5 \mathrm{GeV} / \mathrm{c}$. Here, we make a comparison of this data with the prediction in the current work. In our calculation above, the $\sigma\left(\pi^{-} p \rightarrow f_{1}(1420) n\right) \simeq 0.017(\mu \mathrm{b})$ at $p_{\mathrm{Lab}}=32.5 \mathrm{GeV} / \mathrm{c}$ by taking a value of cutoff $\Lambda=1.6 \mathrm{GeV}$ in the Regge model. For the $K^{-} p \rightarrow f_{1}(1420) \Lambda$ reaction, by taking a

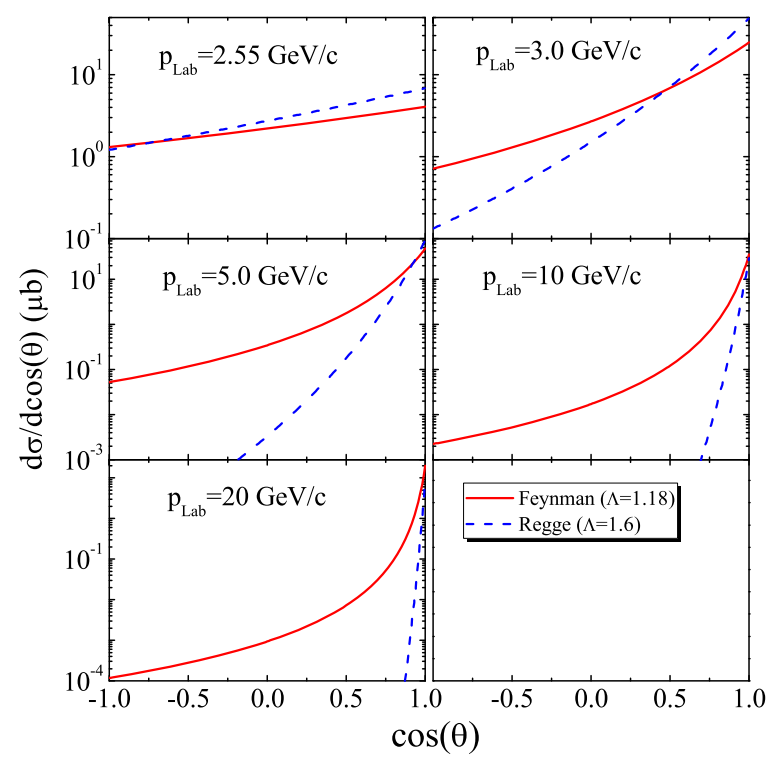

FIG. 3. The differential cross section $d \sigma / d \cos \theta$ of the $\pi^{-} p \rightarrow$ $f_{1}(1420) n$ process as a function of $\cos \theta$. The Full (red) and dashed (blue) lines are for the results of Feynman model and Regge model, respectively.

value of the cutoff $\Lambda=1.6 \mathrm{GeV}$, one can get a value of the total cross section about $0.14 \mu \mathrm{b}$ in the Regge model, which means that the value of the cutoff $\Lambda=1.6 \mathrm{GeV}$ is relatively reasonable to calculate the cross section of the $K^{-} p \rightarrow$ $f_{1}(1420) \Lambda$ reaction in the Regge model. In Fig. 4 we present the total cross section of the $K^{-} p \rightarrow f_{1}(1420) \Lambda$ reaction within the Regge model.

In the Feynman case, if we choose the value of the cutoff as in the pion induced case, the cross section of the $K^{-} p \rightarrow$ $f_{1}(1420) \Lambda$ reaction will increase continuously with the increasing of the momentum $p_{\mathrm{Lab}}$ in the momentum range considered. Though it does not conflict with

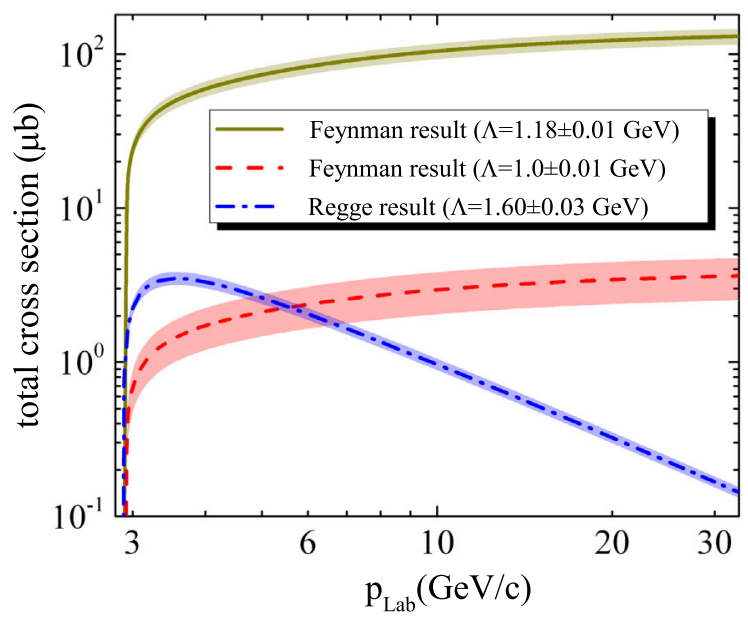

FIG. 4. Total cross section for $K^{-} p \rightarrow f_{1}(1420) \Lambda$ reaction. The Full (red) and dashed (blue) lines are for the Feynman model and Regge model, respectively. The bands stand for the error bar of cutoff $\Lambda_{t}$. 


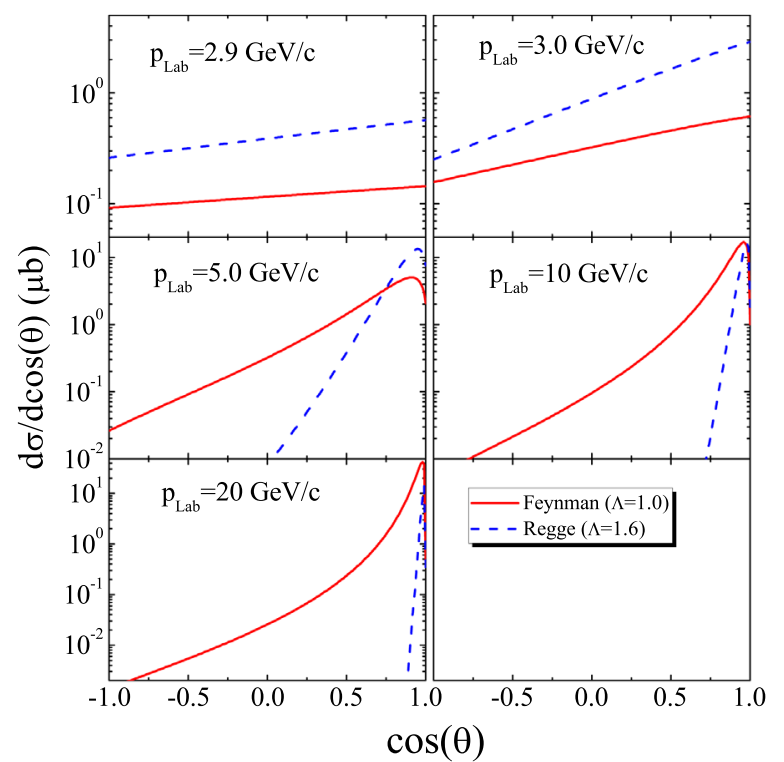

FIG. 5. The differential cross section $d \sigma / d \cos \theta$ of the $K^{-} p \rightarrow$ $f_{1}(1420) \Lambda$ process as a function of $\cos \theta$. The Full (red) and dashed (blue) lines are for the results of Feynman model and Regge model, respectively.

$\sigma\left(K^{-} p \rightarrow f_{1}(1420) \Lambda\right) / \sigma\left(\pi^{-} p \rightarrow f_{1}(1420) n\right)>10$ as suggested in Ref. [11], it seems unnatural that a total cross section will reach $100 \mu \mathrm{b}$. Hence, here we chose a smaller value of cutoff $\Lambda, 1.0 \mathrm{GeV}$.

Different from the pion induced case, from Fig. 4, the line shape of total cross section in the Regge model is quite different from that in the Feynman model. For the Regge case, we notice that the line shape of the total cross section goes up very rapidly and has a peak around $p_{\mathrm{Lab}}=$ $3.53 \mathrm{GeV} / \mathrm{c}$. For the Feynman case, the value of total cross section becomes larger and larger with the increasing of the beam momentum up to $20 \mathrm{GeV} / \mathrm{c}$. The monotonically increasing behavior may be caused by the $K^{*}$ exchange amplitude in the Feynman model [31]. Compared with the Regge model, as discussed in Sec. II C, the Feynman model is less suitable to describe the behavior of the cross section at high beam momenta. The differences between the Regge and the Feynman model will be useful in clarifying the role of the Reggeized treatment in future experiment.

The differential cross sections in the two models are illustrated in Fig. 5, which shows that the discrepancy of the differential cross sections in the two models is small at low beam momenta but becomes large at higher beam momenta. From Fig. 5, one notices that, comparing with the results in the Feynman model, the differential cross section in the Regge model is very sensitive to the $\theta$ angle and gives a considerable contribution at forward angles with the increase of beam momentum.

\section{SUMMARY AND DISCUSSION}

We have studied the $\pi^{-} p \rightarrow f_{1}(1420) n$ and $K^{-} p \rightarrow$ $f_{1}(1420) \Lambda$ reactions within the Feynman and Regge models. For the $\pi^{-} p \rightarrow f_{1}(1420) n$ reaction, both results calculated in the Regge and the Feynman models can be reproduced in the experimental data, but the differential cross sections at high beam momenta are quiet different. It is found that the differential cross sections for the $\pi^{-} p \rightarrow$ $f_{1}(1420) n$ reaction in the Regge model are very sensitive to the $\theta$ angle and give considerable contributions at forward angles, which can be checked by future experiment and may be an effective way to examine the validity of the Reggeized treatment.

For the $K^{-} p \rightarrow f_{1}(1420) \Lambda$ reaction, the line shapes of the total cross sections obtained in both models are very different. The total cross section increases continuously in the Feynman model in the beam momentum range considered in the current work. If the cutoff of the pion induced production is adopted directly, the cross section increases to $100 \mu$ b. In the Regge model, it decreases at momenta larger than $p_{\text {Lab }}=4 \mathrm{GeV} / \mathrm{c}$. It is consistent with the Regge model being more suitable to describe the behavior of the cross section at high momenta. The line shape of the differential cross section of the $K^{-} p \rightarrow f_{1}(1420) \Lambda$ reaction is similar to the result of the $\pi^{-} p \rightarrow f_{1}(1420) n$ reaction. In the Regge model, the $t$ channel provides a sharp increase at extreme forward angles.

In the current work, the pion induced $f_{1}(1420)$ production is studied with the $a_{0}$ exchange (see Fig. 1). The results suggest that the couplings of the $f_{1}(1420)$ with $a_{0} \pi$ should be considerably large. To some extent, it supports the interpretation of the $f_{1}(1420)$ as a reflection of the decay modes of the $f_{1}(1285)$ in Ref. [18] where a large branch ratio, about $17 \%$, in the $a_{0} \pi$ channel is extracted. Of coarse, it does not mean that interpretations in the $s \bar{s}$ picture are disfavored, because there is no theoretical calculation about the $a_{0} \pi$ decay in the constituent quark model. To deepen the understanding of the $f_{1}(1420)$, experimental studies of the $f_{1}(1420)$ with the pion and kaon beams are strongly suggested. The results of this work suggest that it is promising to do such an experiment at existing facilities. The expected high-precision data will be very helpful to extract the couplings of the $f_{1}(1420)$ and $K^{*} \bar{K}$ and $a_{0} \pi$ with the method in this work. Besides, the subsequential decay that produced $f_{1}(1420)$ in such experiments will provide more information about the $f_{1}(1420)$.

The pion and kaon beams can be provided at J-PARC and COMPASS. The precision of possible data in future experiments at these facilities will be much higher than in old experiments. The above theoretical results may provide valuable information for possible experiments to study the $f_{1}(1420)$ at these facilities. 


\section{ACKNOWLEDGMENTS}

This project is supported by the National Natural Science Foundation of China under Grants No. 11705076 and No. 11675228. We acknowledge the Natural Science Foundation of Gansu province under Grant No. 17JR5RA113.

[1] M. Tanabashi et al. (Particle Data Group), Review of particle physics, Phys. Rev. D 98, 030001 (2018).

[2] A. Austregesilo (GlueX Collaboration), Light-meson spectroscopy at GlueX, Int. J. Mod. Phys. 46, 1860029 (2018).

[3] S. Kumano, Spin physics at J-PARC, Int. J. Mod. Phys. 40, 1660009 (2016).

[4] F. Nerling (COMPASS Collaboration), Hadron spectroscopy with COMPASS: Newest results, Eur. Phys. J. Web Conf. 37, 01016 (2012).

[5] V. Obraztsov (OKA Collaboration), High statistics measurement of the $K^{+} \rightarrow \pi^{0} e^{+} \nu(\mathrm{Ke} 3)$ decay formfactors, Nucl. Part. Phys. Proc. 273-275, 1330 (2016).

[6] B. Velghe (NA62-RK and NA48/2 Collaborations), $K^{ \pm} \rightarrow$ $\pi^{ \pm} \gamma \gamma$ studies at NA48/2 and NA62-RK experiments at CERN, Nucl. Part. Phys. Proc. 273-275, 2720 (2016).

[7] T. Nagae, The J-PARC project, Nucl. Phys. A805, 486c (2008).

[8] O. I. Dahl, L. M. Hardy, R. I. Hess, J. Kirz, and D. H. Miller, Strange-particle production in $\pi p$ interactions from 1.5 to 4.2 BeV/c. 1. Three-and-more-body final states, Phys. Rev. 163, 1377 (1967).

[9] M. J. Corden et al., Observation of the $D, e$ and delta mesons in $\pi^{-} p$ interactions at $12-\mathrm{GeV} / c$ and $15-\mathrm{GeV} / c$, Nucl. Phys. B144, 253 (1978).

[10] C. Dionisi et al. (CERN-College de France-MadridStockholm Collaboration), Observation and quantum numbers determination of the $\mathrm{E}(1420)$ meson in $\pi^{-} p$ interactions at 3.95-GeV/c, Nucl. Phys. B169, 1 (1980).

[11] S. I. Bityukov et al., Investigation of $D(1285)$ and $e(1420)$ mesons production in exclusive interactions of $\pi^{-}$and $K^{-}$ mesons at 32.5-GeV/c, Sov. J. Nucl. Phys. 39, 735 (1984).

[12] J. Abdallah et al. (DELPHI Collaboration), Measurement of inclusive $f_{1}(1285)$ and $f_{1}(1420)$ production in $\mathrm{Z}$ decays with the DELPHI detector, Phys. Lett. B 569, 129 (2003).

[13] P. Achard et al. (L3 Collaboration), Study of resonance formation in the mass region $1400-\mathrm{MeV}$ to $1500-\mathrm{MeV}$ through the reaction $\gamma \gamma \rightarrow K_{s}^{0} K^{ \pm} \pi^{\mp}$, J. High Energy Phys. 03 (2007) 018.

[14] Z. Bai et al. (MARK-III Collaboration), Partial Wave Analysis of $J / \psi \rightarrow \gamma K_{s}^{0} K^{ \pm} \pi^{\mp}$, Phys. Rev. Lett. 65, 2507 (1990).

[15] X. Liu, Z. J. Xiao, and Z. T. Zou, Nonleptonic decays of $B \rightarrow\left(f_{1}(1285), f_{1}(1420)\right) V$ in the perturbative QCD approach, Phys. Rev. D 94, 113005 (2016).

[16] F. E. Close and A. Kirk, Implications of the glueball $q \bar{q}$ filter on the $1^{++}$nonet, Z. Phys. C 76, 469 (1997).

[17] D. M. Li, H. Yu, and Q. X. Shen, Is f(1)(1420) the partner of $f_{1}(1285)$ in the ${ }^{3} P_{1} q \bar{q}$ nonet?, Chin. Phys. Lett. 17, 558 (2000).
[18] V. R. Debastiani, F. Aceti, W. H. Liang, and E. Oset, Revising the $f_{1}(1420)$ resonance, Phys. Rev. D 95, 034015 (2017).

[19] X. Y. Wang and J. He, Investigation of pion-induced $f_{1}(1285)$ production off a nucleon target within an interpolating Reggeized approach, Phys. Rev. D 96, 034017 (2017).

[20] D. Barberis et al. (WA102 Collaboration), A measurement of the branching fractions of the $f_{1}(1285)$ and $f_{1}(1420)$ produced in central $p p$ interactions at $450-\mathrm{GeV} / \mathrm{c}$, Phys. Lett. B 440, 225 (1998).

[21] N. I. Kochelev, M. Battaglieri, and R. De Vita, Exclusive photoproduction of $f_{1}(1285)$ meson off proton in the JLab kinematics, Phys. Rev. C 80, 025201 (2009).

[22] S. K. Domokos, H. R. Grigoryan, and J. A. Harvey, Photoproduction through Chern-Simons term induced interactions in holographic QCD, Phys. Rev. D 80, 115018 (2009).

[23] X. H. Liu, Q. Zhao, and F. E. Close, Search for tetraquark candidate Z(4430) in meson photoproduction, Phys. Rev. D 77, 094005 (2008).

[24] P. Colangelo and F. De Fazio, Open charm meson spectroscopy: Where to place the latest piece of the puzzle, Phys. Rev. D 81, 094001 (2010).

[25] X. Y. Wang and J. He, $K^{* 0} \Lambda$ photoproduction off a neutron, Phys. Rev. C 93, 035202 (2016).

[26] X. Y. Wang and X. R. Chen, Production of the superheavy baryon $\Lambda_{c \bar{c}}^{*}(4209)$ in kaon-induced reaction, Eur. Phys. J. A 51, 85 (2015).

[27] V. G. J. Stoks and T. A. Rijken, Soft-core baryon-baryon potentials for the complete baryon octet, Phys. Rev. C 59, 3009 (1999).

[28] H. Haberzettl, X. Y. Wang, and J. He, Preserving local gauge invariance with t-channel Regge exchange, Phys. Rev. C 92, 055503 (2015).

[29] X. Y. Wang, J. He, and H. Haberzettl, Analysis of recent CLAS data on $\Sigma^{*}(1385)$ photoproduction off a neutron target, Phys. Rev. C 93, 045204 (2016).

[30] G. Galata, Photoproduction of Z(4430) through mesonic Regge trajectories exchange, Phys. Rev. C 83, 065203 (2011).

[31] S. Ozaki, H. Nagahiro, and A. Hosaka, Charged K* photoproduction in a Regge model, Phys. Rev. C 81, 035206 (2010).

[32] X. Y. Wang and J. He, Analysis of recent CLAS data on $f_{1}(1285)$ photoproduction, Phys. Rev. D 95, 094005 (2017).

[33] S. i. Nam and C. W. Kao, $\Lambda(1520)$ photoproduciton off the proton target with Regge contributions, Phys. Rev. C 81, 055206 (2010). 